\title{
On nestedly complete topological vector lattices
}

\author{
Lech Drewnowski
}

Received: 5 February 2010 / Accepted: 1 July 2010 / Published online: 5 August 2010 C The Author(s) 2010. This article is published with open access at Springerlink.com

\begin{abstract}
A topological vector lattice $E$ is called ( $\sigma$-)nestedly complete if every downward directed net (resp., decreasing sequence) of order intervals in $E$ whose 'diameters' tend to zero has a nonempty intersection. Some characterizations of the $(\sigma-)$ nested completeness are given, and it is shown that if $E$ is metrizable and nestedly complete, so is each of its quotients $E / I$, where $I$ is a closed ideal in $E$. Conversely, if a closed ideal $I$ in $E$ is (sequentially) complete and $E / I$ is $(\sigma$-)nestedly complete, so is $E$. However, the nested completeness is not a three-space property: an example is given where both $I$ and $E / I$ are nestedly complete while $E$ is not. It is also shown that the nested completeness and the related notion of nested density come up quite naturally when extending some positive linear operators. Finally, the nested and other completeness type properties of vector lattices $C(S)$ are investigated.
\end{abstract}

Keywords Topological vector lattice - Nested completeness - Quotient vector lattice · Three space property · Nested density · Extension of positive operators · Nested completion

Mathematics Subject Classification (2000) $\quad 46 \mathrm{~A} 16 \cdot 46 \mathrm{~A} 40 \cdot 28 \mathrm{~A} 12 \cdot$ $54 \mathrm{D} 05 \cdot 54 \mathrm{~F} 15 \cdot 54 \mathrm{H} 11 \cdot 54 \mathrm{H} 12$

To Professor Wilhelmus A. J. Luxemburg on his 80th birthday.

This research was partially supported by The Ministry of Science and Higher Education, Poland, Grant no. N N 201274033.

L. Drewnowski $(\varangle)$

Faculty of Mathematics and Computer Science, A. Mickiewicz University,

Umultowska 87, 61-614 Poznań, Poland

e-mail: drewlech@amu.edu.pl 


\section{Introduction and main concepts}

For the sake of clarity, we start with a brief review of some standard notions and facts. Let $E=(E, \tau)$ be a (Hausdorff) topological vector space (TVS) with a base $\mathcal{U}$ at zero. A filter base in $E$ is a nonempty family $\mathcal{B}$ consisting of nonempty subsets of $E$ which is directed downward: for any $B_{1}, B_{2} \in \mathcal{B}$ there is $B_{3} \in \mathcal{B}$ with $B_{3} \subset B_{1} \cap B_{2}$. It is Cauchy if it contains sets $B$ of arbitrarily small 'diameter' $B-B$, that is, for every $U \in \mathcal{U}$ there is $B \in \mathcal{B}$ with $B-B \subset U$; and convergent to a point $z \in E$ if for every $U \in \mathcal{U}$ there is $B \in \mathcal{B}$ with $B \subset z+U$. The space $E$ is complete, (C), if every Cauchy filter base in $E$ converges.

More generally, by specifying a class of Cauchy filter bases that are required to converge, one gets the corresponding variant of the notion of completeness. Trivially, the smaller is the class, the weaker is the resulting type of completeness. Let us mention two important special cases.

If the filter bases involved are all those that consist of bounded sets (or just contain a bounded set), the result is the bounded completeness, (b-C); it is usually called quasi-completeness.

For the class of all countable Cauchy filter bases, the resulting notion is that of $\sigma$-completeness, $\sigma-(\mathrm{C})$. Note that every countable filter base $\mathcal{B}$ contains a $\sigma$-filter base, i.e., a decreasing sequence of sets $\mathcal{B}^{\prime}=\left(B_{n}\right)_{n \in \mathbb{N}}$, such that each $B \in \mathcal{B}$ contains one of the $B_{n}$ 's. Then, clearly, $\mathcal{B}$ is Cauchy (or convergent to a point $z$ ) iff so is $\mathcal{B}^{\prime}$. It follows that $E$ is $\sigma$-complete iff every Cauchy $\sigma$-filter base in $E$ converges, and the latter property is easily seen to be the same as the usual sequential completeness of $E$. Therefore, $(\mathrm{C}) \Longrightarrow(\mathrm{b}-\mathrm{C}) \Longrightarrow \sigma-(\mathrm{C})$, and the ' $\sigma$-version' $\sigma-(\mathrm{b}-\mathrm{C})$ of $(\mathrm{b}-\mathrm{C})$ that one might think of would coincide with $\sigma-(\mathrm{C})$.

If the space $E$ is metrizable, then every filter base in $E$ that is Cauchy (or convergent to a point $z$ ) contains a $\sigma$-filter base with the same property. Hence in this case $(\mathrm{C})$, (b-C), and $\sigma-(\mathrm{C})$ coincide.

Let now $E=(E, \tau)$ be a (Hausdorff locally solid) topological vector lattice (TVL). In this case, by replacing bounded sets by order bounded sets, one arrives at natural (and of course weaker) order-variants of (b-C) and $\sigma-(\mathrm{b}-\mathrm{C})=\sigma-(\mathrm{C})$, namely, the orderbounded completeness, (ob-C), and its $\sigma$-version, $\sigma$-(ob-C). They are known in the literature as the interval completeness and $\sigma$-interval completeness, and simply mean that every order interval in $E$ is complete or sequentially complete, respectively. (See e.g. [10] and [1], and also [2], Theorems 2.42, 2.46, 4.28.) In this paper, we consider a still weaker concept of completeness for a TVL, the nested completeness along with its $\sigma$-variant, the $\sigma$-nested completeness.

By a $(\sigma-)$ nest in $E$ we shall mean any $(\sigma-)$ filter base in $E$ consisting of order intervals. We shall say that the TVL $E$ is $(\sigma$-)nestedly complete if every Cauchy (countable or just $\sigma_{-}^{-}$) nest in $E$ is convergent. Obviously, a classic model for these concepts is the property of the real line described by the nested interval theorem (or the Ascoli's lemma) from the elementary analysis. Let us remark that the concept of $\sigma$-nested completeness, in a more general context, has recently been used in [5]. We continue with some explanations.

Let $\mathcal{B}$ be a nest in $E$. As is often convenient, let us view $\mathcal{B}$ as a net $\left(\left[x_{\alpha}, y_{\alpha}\right]\right)_{\alpha \in A}$ of order intervals, directed downward by inclusion. Then the nets $\left(x_{\alpha}\right)$ and $\left(y_{\alpha}\right)$ are 
directed upward and downward, respectively, with $x_{\alpha} \leqslant y_{\beta}$ whenever $\alpha, \beta \in A$ and $\alpha \leqslant \beta$. (In particular, these nets are order bounded from above and below, respectively.) For an order interval $[x, y]$ in $E$, one has $|u-v| \leqslant y-x$ whenever $u, v \in[x, y]$; thus the 'diameter' $[x, y]-[x, y]$ of the interval is 'small' iff its 'length' $y-x$ is 'small'. Hence the nest $\mathcal{B}$ is Cauchy iff $y_{\alpha}-x_{\alpha} \rightarrow 0$. (In particular, every net $\left(z_{\alpha}\right)$ with $z_{\alpha} \in\left[x_{\alpha}, y_{\alpha}\right]$ for each $\alpha$ is then Cauchy.) Next, the nest $\mathcal{B}$ converges to a point $z \in E\left(z=\lim \mathcal{B}=\lim _{\alpha}\left[x_{\alpha}, y_{\alpha}\right]\right)$ iff $z=\lim _{\alpha} x_{\alpha}=\lim _{\alpha} y_{\alpha}$ (and then one also has $z=\sup _{\alpha} x_{\alpha}=\inf _{\alpha} y_{\alpha}$ ). Finally, since the order intervals are closed, if the nest $\mathcal{B}$ is Cauchy, then it converges (to some $z \in E$ ) iff $\mathcal{B}$ has a nonempty intersection (which is then necessarily the singleton $\{z\}$ ) iff $\lim _{\alpha} x_{\alpha}$, or $\lim _{\alpha} y_{\alpha}$, or $\sup _{\alpha} x_{\alpha}$, or inf $y_{\alpha} y_{\alpha}$ exists in $E$.

Let us proceed with a few simple observations. If $E$ is metrizable, then it is nestedly complete iff it is $\sigma$-nestedly complete. If $E$ is $(\sigma$-)Dedekind complete or $(\sigma$-)intervally complete, then it is $(\sigma-)$ nestedly complete. (However, as we will see in Sect. 5, there exist nestedly complete normed lattices that are neither $\sigma$-Dedekind complete nor intervally complete.) If $E$ is $(\sigma$-)nestedly complete, so are all of its ideals and, more generally, all of its $(\sigma-)$ nestedly closed (see below) vector sublattices. In consequence, $E$ is $(\sigma-)$ nestedly complete iff it is $(\sigma$-)nestedly closed in the topological completion $\tilde{E}$ of $E$. In contrast to the other types of completeness, the $(\sigma-)$ nested completeness of $E$ is preserved when passing to any stronger locally solid topology. Finally, the product of any family of ( $\sigma$-)nestedly complete TVL's is also $(\sigma-)$ nestedly complete. (The case of quotients is discussed in Sect. 3) From what was said above it follows that, e.g., the vector lattices $\ell_{p}(0<p \leqslant \infty)$ and $c_{0}$ are nestedly complete in the topology of coordinate-wise convergence, while $c$ is not, as easily seen. Likewise, if $\mu$ is a finite measure, then $L_{p}(\mu)(0<p \leqslant \infty)$ is nestedly complete in the topology of convergence in measure.

Let us also note that the $\sigma$-nested completeness is strictly weaker than the $\sigma$-interpolation property of vector lattices (see [11, Ch. 20, §146]). (The latter requires every $\sigma$-nest to have a nonempty intersection.) E.g., the Banach lattice $C[0,1]$ is (trivially) nestedly complete but does not have the $\sigma$-interpolation property.

One may think of a convergent nest as providing arbitrarily close approximations to its limit, simultaneously from below and from above. Following this point of view, we define the nested closure of a vector sublattice $L$ of the TVL $E$ to be the set $M$ of all those points in $E$ that can be obtained as the limit of a convergent nest of order intervals in $E$ whose end-points are in $L$. As expected, $M$ is again a vector sublattice of $E$ (see Proposition 4.1), and we shall say that $L$ is nestedly closed in $E$ if $M=L$, and nestedly dense in $E$ if $M=E$. (Some examples of nestedly dense sublattices are given in Sect. 4) Also, we shall say that a positive linear operator $T$ from $E$ to a TVL $F$ is nestedly continuous if, for every Cauchy nest $\left(\left[x_{\alpha}, y_{\alpha}\right]\right)$ in $E$, the nest $\left(\left[T x_{\alpha}, T y_{\alpha}\right]\right)$ is Cauchy in $F$. Equivalently, whenever $0 \leqslant z_{\alpha} \downarrow$ and $z_{\alpha} \rightarrow 0$ in $E$, then $\left(0 \leqslant T z_{\alpha} \downarrow\right.$ and) $T z_{\alpha} \rightarrow 0$ in $F$. (The concept of nested continuity is also meaningful in other 'admissible' contexts.) Replacing nests with $\sigma$-nests, we get the $\sigma$-versions of these concepts. Again, it is not hard to verify that if $E$ is metrizable, then the general versions coincide with their $\sigma$-counterparts.

In the paper, we first give some restatements of the definition of $(\sigma$-)nested completeness of the TVL $E$ (Proposition 2.1), and a characterization of the $\sigma$-nested 
completeness of $E$ in terms of extendability of $E$-valued uniformly continuous increasing functions (Proposition 2.3). Next, we show that if $E$ is metrizable and nestedly complete, so are its quotients $E / I$, where $I$ is any closed ideal in $E$ (Theorem 3.2). Conversely, we prove in Theorem 3.4 that, for a general TVL $E$, if $I$ is $(\sigma-)$ nestedly complete in $E$ (see the paragraph right before that theorem), and $E / I$ is $(\sigma-)$ nestedly complete, so is $E$. However, the nested completeness is not a threespace property (Example 3.6). We also prove that a nestedly continuous positive linear operator $T$ from a nestedly dense sublattice $L$ of $E$ to a nestedly complete TVL $F$ has always a like extension $\tilde{T}: E \rightarrow F$ (Theorem 4.5). A consequence of this is worth mentioning: every nestedly continuous positive linear operator defined on a nestedly dense sublattice of an $F$-lattice is continuous (Corollary 4.7). Then the concept of nested completion is discussed shortly. Finally, in Sect. 5, we investigate the presence (or lack) of complete nontrivial order intervals in the vector lattices $C(S)$ of bounded continuous functions, equipped with various locally solid topologies $\tau$. Here it is important that $(C(S), \tau)$ is nestedly complete whenever $\tau$ is finer than the topology of pointwise convergence (Proposition 5.1).

Our terminology is rather standard, though slightly more neutral than that in $[2,3]$ or [11], for instance. Thus we deal with topological vector lattices (or Hausdorff locally solid Riesz spaces), $F$-lattices (complete metrizable topological vector lattices), and Banach lattices. A vector (sub)lattice is the same as a Riesz (sub)space, and a (vector) lattice homomorphism is the same as a Riesz homomorphism. We use the commonly accepted notation for the Banach lattices $\ell_{p}$ and $L_{p}(\mu)(1 \leqslant p<\infty$, with the norm $\|\cdot\|_{p}$ ); $c$ and $c_{0}$ (with the supnorm $\|\cdot\|_{\infty}$ ); and $p$-Banach lattices $\ell_{p}$ and $L_{p}(\mu)$ $\left(0<p<1\right.$, with the $p$-norm $\left.\|\cdot\|_{p}\right)$.

\section{Characterizations of nested completeness}

Let $A$ and $B$ be nonempty subsets of a TVL $E$ such that $A \leqslant B$ (that is, $x \leqslant y$ whenever $x \in A, y \in B$ ), and let us say that there is no (topological) gap between $A$ and $B$ if for every neighborhood $U$ of zero there are $x \in A$ and $y \in B$ with $y-x \in U$. If this is the case, then one is inclined to expect that $\sup A=\inf B$ exist in $E$, and the proposition below shows that it is indeed so iff $E$ is nestedly complete. In other words, the nested completeness can be viewed as a 'topologically spiced' version of the Dedekind completeness.

Proposition 2.1 Let $E$ be a TVL.

(a) $E$ is nestedly complete iff the following condition is satisfied: Whenever $A$ is a nonempty subset of $E$ which is order bounded from above, $B$ is the set of upper bounds for $A$, and there is no gap between $A$ and $B$, then sup A exists in $E$.

(b) $E$ is $\sigma$-nestedly complete iff the following condition is satisfied: Whenever $A$ and $B$ are as in (a), and there exist sequences $\left(x_{n}\right)$ in $A$ and $\left(y_{n}\right)$ in $B$ with $y_{n}-x_{n} \rightarrow 0$, then $\sup A$ exists in $E$.

Proof (a) 'Only if.' For $A$ and $B$ as specified above, one may also assume that $A$ is directed upward. Then the net $([x, y]: x \in A, y \in B)$ is a Cauchy nest. Therefore, it has a limit in $E$ which is also the sup of $A$. 
'If.' Consider any Cauchy nest $\mathcal{B}$ in $E$, and apply the condition to the set $A$ consisting of the left end-points of the intervals in $\mathcal{B}$.

(b) 'Only if.' Again, we may assume that $A$ is directed upward. For every $n$, let $x_{n}^{\prime}=x_{1} \vee \cdots \vee x_{n}$ and $y_{n}^{\prime}=y_{1} \wedge \cdots \wedge y_{n}$. Then $\left(x_{n}^{\prime}\right) \subset A,\left(y_{n}^{\prime}\right) \subset B, x_{n}^{\prime} \uparrow, y_{n}^{\prime} \downarrow$ and $x_{m} \leqslant x_{m}^{\prime} \leqslant y_{n}^{\prime} \leqslant y_{n}$ for all $m, n$. It follows that the sequence $\left(\left[x_{n}^{\prime}, y_{n}^{\prime}\right]\right)$ is a Cauchy $\sigma$-nest. Therefore, it has a limit in $E$ which is also the sup of $A$.

'If.' Like for the 'if' case above.

Corollary 2.2 If a TVL $E$ is nestedly complete and has the Lebesgue property (that is, $x_{\alpha} \rightarrow 0$ whenever $\left.x_{\alpha} \downarrow 0\right)$, then it is Dedekind complete.

Proof Let $A$ be a nonempty subset of $E$ that is order bounded from above, and let $B$ stand for the set of the upper bounds for $A$. Assume, as we may, that $A$ is directed upward, Then the net $(y-x: x \in A, y \in B)$ is directed downward and has 0 as its infimum. Therefore, it converges to 0 so that the condition required in part (a) of the proposition above is satisfied. Hence sup $A$ exists in $E$.

Proposition 2.3 Let $E$ be a TVL, and let $D$ be a proper dense subset of the interval $[0,1]$ in $\mathbb{R}$ containing its endpoints. Then $E$ is $\sigma$-nestedly complete iff every uniformly (or nestedly) continuous increasing function $\varphi: D \rightarrow E$ has an extension to a continuous incresing function $\tilde{\varphi}:[0,1] \rightarrow E$.

Proof 'Only if'. For each $t \in[0,1]$, choose an increasing sequence $\left(s_{n}\right)$ in $D$, and a decreasing sequence $\left(s_{n}^{\prime}\right)$ in $D$, both with limit $t$. Then the sequence $\left(\left[\varphi\left(s_{n}\right), \varphi\left(s_{n}^{\prime}\right)\right]\right)$ is a Cauchy $\sigma$-nest in $E$. By the $\sigma$-nested completeness of $E$, it has a limit $\tilde{\varphi}(t)$ in $E$. As easily seen, $\tilde{\varphi}(t)=\sup \{\varphi(s): s \in D \& s \leqslant t\}=\inf \left\{\varphi\left(s^{\prime}\right): s^{\prime} \in D \& t \leqslant s^{\prime}\right\}$. Using this along with the uniform (or nested) continuity of $\varphi$ it is not hard to verify that $\tilde{\varphi}$ is a required extension of $\varphi$.

'If'. Let $\left(\left[x_{n}, y_{n}\right]\right)_{n \in \mathbb{N}}$ be a Cauchy $\sigma$-nest in $E$. We may assume that the sequences $\left(x_{n}\right)$ and $\left(y_{n}\right)$ are strictly increasing and strictly decreasing, respectively. Fix any point $t_{0} \in(0,1) \backslash D$, and choose sequences $0=s_{1}<s_{2}<\ldots$ and $1=s_{1}^{\prime}>s_{2}^{\prime}>\ldots$ in $D$, both with limit $t_{0}$. Then, for each $n \in \mathbb{N}$, let $\varphi\left(s_{n}\right)=x_{n}$ and $\varphi\left(s_{n}^{\prime}\right)=y_{n}$, and extend $\varphi$ by linearity to each of the subintervals $\left[s_{n}, s_{n+1}\right)$ and $\left(s_{n+1}^{\prime}, s_{n}^{\prime}\right]$ of $[0,1]$. The function $\varphi$ thus defined on $[0,1] \backslash\left\{t_{0}\right\}$ is easily seen to be increasing and uniformly continuous (and so is of course $\varphi \mid D$ ). By assumption, it can be extended to a similar function $\tilde{\varphi}:[0,1] \rightarrow E$. Then one has $\tilde{\varphi}\left(t_{0}\right)=\lim _{n} x_{n}=\lim _{n} y_{n}$, and the proof is complete.

\section{Quotients of nestedly complete TVL's}

In all of this section, $E$ is a TVL, $I$ is a closed ideal in $E$, and $Q: E \rightarrow E / I$ is the corresponding quotient lattice (or Riesz) homomorphism. Whenever $E$ is assumed to be metrizable, we let $\|\cdot\|$ denote a monotone $F$-norm defining its topology.

Lemma 3.1 Let $[u, v]$ be an order interval in $E / I$.

(a) There exist $x, y \in E$ such that $x \leqslant y, Q x=u$ and $Q y=v$. 
(b) Whenever $x, y \in E$ are such that $x \leqslant y, Q x=u$ and $Q y=v$, then $Q([x, y])=$ $[u, v]$. Moreover, if $E$ is metrizable, then for every $w \in[u, v]$ and any $\varepsilon>0$ there is $z \in[x, y]$ such that $Q z=w$ and $\|z-x\| \leqslant\|w-u\|+\varepsilon$.

Proof (a) Take any $x, z \in E$ with $Q x=u, Q z=v$. Then $x$ and $y:=x \vee z$ are as required.

(b) Let $w \in[u, v]$. Choose any $b \in E$ with $Q b=w$, and set $z=(x \vee b) \wedge y$. Then $z \in[x, y]$ and $Q z=(Q x \vee Q b) \wedge Q y=(u \vee w) \wedge v=w$. To prove the 'moreover' part, select any $a \in E$ with $Q a=w-u$ and $\|a\| \leqslant\|w-u\|+\varepsilon$. Then also $Q\left(a^{+}\right)=w-u$ and $\left\|a^{+}\right\| \leqslant\|a\|$. Let $z=\left(x+a^{+}\right) \wedge y$. Then $z \in[x, y]$, $Q z=\left(Q x+Q a^{+}\right) \wedge Q y=w$, and $\|z-x\|=\left\|a^{+} \wedge(y-x)\right\| \leqslant\left\|a^{+}\right\|$.

Theorem 3.2 If E is metrizable and nestedly complete, so is the quotient E/I.

Proof Below, we make a repeated use of the preceding lemma.

Let $\left(\left[u_{n}, v_{n}\right]\right)_{n \in \mathbb{N}}$ be a Cauchy $\sigma$-nest in $E / I$. Choose any $x_{1}, y_{1} \in E$ with $x_{1} \leqslant y_{1}$ so that $Q x_{1}=u_{1}$ and $Q y_{1}=v_{1}$. Since $Q\left(\left[x_{1}, y_{1}\right]\right)=\left[u_{1}, v_{1}\right]$, there is $x_{2} \in\left[x_{1}, y_{1}\right]$ with $Q x_{2}=u_{2}$. Now, $Q\left(\left[x_{2}, y_{1}\right]\right)=\left[u_{2}, v_{1}\right]$, hence there is $y_{2} \in\left[x_{2}, y_{1}\right]$ such that $Q y_{2}=v_{2}$ and $\left\|y_{2}-x_{2}\right\| \leqslant\left\|v_{2}-u_{2}\right\|+\frac{1}{2}$.

We continue in an obvious manner to produce a decreasing sequence $\left(\left[x_{n}, y_{n}\right]\right)$ of intervals in $E$ such that $Q x_{n}=u_{n}$ and $Q y_{n}=v_{n}$ for each $n$, and $\left\|y_{n}-x_{n}\right\| \leqslant$ $\left\|v_{n}-u_{n}\right\|+\frac{1}{n}$ for $n \geqslant 2$. Since $E$ is nestedly complete, the intersection of these intervals is a singleton, $\{z\}$ say, and then $Q z$ is in each of the intervals $\left[u_{n}, v_{n}\right]$.

Question 3.3 Is the metrizability of $E$ essential? (Conjecture: It is!)

For the purpose of our next result, let us say that the ideal $I$ is $(\sigma$-)nestedly complete in $E$ if every Cauchy ( $\sigma$-)nest in $E$ all of whose members intersect $I$ is convergent (to a point in $I$, of course). Clearly, if $E$ is ( $\sigma$-)nestedly complete, then $I$ is $(\sigma$-)nestedly complete in $E$, and the latter implies that $I$ is $(\sigma-)$ nestedly complete (in itself). Also note that if the ideal $I$ is (sequentially) complete, then it is $(\sigma-)$ nestedly complete in E. (See also Remark 3.5 below.)

Theorem 3.4 If the ideal I is $(\sigma$-)nestedly complete in $E$ and the quotient $E / I$ is $(\sigma-)$ nestedly complete, then $E$ is $(\sigma-)$ nestedly complete.

Prooffor the $\sigma$-case Let $\left(\left[x_{n}, y_{n}\right]\right)_{n \in \mathbb{N}}$ be a Cauchy $\sigma$-nest in $E$. Then the sequence $\left(\left[u_{n}, v_{n}\right]\right)_{n \in \mathbb{N}} \equiv\left(Q\left(\left[x_{n}, y_{n}\right]\right)\right)_{n \in \mathbb{N}}$ is a Cauchy $\sigma$-nest in $E / I$, and has a limit, $w$ say. Select any point $z \in E$ with $Q z=w$. Then the sequence $\left(\left[x_{n}-z, y_{n}-z\right]\right)_{n \in \mathbb{N}}$ is a Cauchy $\sigma$-nest in $E$ and $\left[x_{n}-z, y_{n}-z\right] \cap I \neq \emptyset$ for every $n$. Hence, by the assumption on $I$, there is $a \in I$ such that $a \in\left[x_{n}-z, y_{n}-z\right]$, or $a+z \in\left[x_{n}, y_{n}\right]$, for every $n$. This completes the proof.

Remark 3.5 Evidently, the ideal $I$ is $(\sigma$-)nestedly complete in $E$ provided that it is $(\sigma-)$ nestedly complete and has the following property: The intersection with $I$ of any order interval in $E$, if nonempty, is an order interval. Equivalently (as easily seen), for any $z \in E_{+}$, the intersection $[0, z] \cap I$ is an order interval. The latter condition on $I$ relative to $E$ is known to coincide with the requirement that $I$ is a projection band 
in $E$ (cf. [3, Th. 1.41]). Now, in that case, $E=I \oplus I^{d}$ and $I^{d}$ is order-topologically isomorphic to $E / I$ so that the resulting particular instance of the theorem would be obvious (and thus not too interesting).

One may rightly wonder if Theorem 3.4 could be improved so as to state that the $(\sigma-)$ nested completeness is a three-space property in the sense that whenever both $I$ and $E / I$ are $(\sigma-)$ nestedly complete, so is $E$. In the example below it is shown that such a refinement of the theorem is impossible. (For more information on the three-space properties in the context of Banach spaces, see e.g. [4].)

Example 3.6 E may fail to be nestedly complete even if $I$ is intervally complete and $E / I$ is of dimension one (hence complete). (Then, of course, $I$ is not nestedly complete in $E$.)

Let $E$ denote the vector lattice of all eventually constant real sequences $x=\left(\xi_{j}\right)$ equipped with the supnorm $\|\cdot\|_{\infty}$. Then its closed ideal $I$ consisting of eventually zero sequences is intervally complete because every order interval in $I$ is finite dimensional. Since $\operatorname{dim} E / I=1, E / I$ is complete. However, $E$ is not nestedly complete. To verify this statement, let $x_{n}=\left(1, \frac{1}{2}, \ldots, \frac{1}{n}, 0,0, \ldots\right)$ and $y_{n}=\left(1, \frac{1}{2}, \ldots, \frac{1}{n}, \frac{1}{n}, \frac{1}{n}, \ldots\right)$ $(n \in \mathbb{N})$. Then the order intervals $\left[x_{n}, y_{n}\right]$ in $E$ form a decreasing sequence and $\left\|y_{n}-x_{n}\right\|_{\infty}=\frac{1}{n} \rightarrow 0$, but their intersection is empty.

\section{Nested completeness and extensions of positive operators}

Let us start by observing that, for a vector sublattice $L$ of a TVL $E$, its nested closure $M$ (as defined in Sect. 1), is precisely the set of all those $x \in E$ such that for every neighborhood $U$ of zero in $E$ there are $u, v \in L$ with $u \leqslant x \leqslant v$ and $v-u \in U$. That is, both the sets $A_{x}=\{u \in L: u \leqslant x\}$ and $B_{x}=\{v \in L: x \leqslant v\}$ are nonempty and there is no 'gap' between them (see the beginning of Sect. 2). Also note that $L$ is nestedly dense in $E$ iff every element of $E$ can be approximated arbitrarily close from above [alternatively, from below] by elements from $L$.

Proposition 4.1 Let $L$ be a vector sublattice of a TVL E. Then the nested closure $M$ of $L$ in $E$ is the smallest nestedly closed vector sublattice of $E$ containing $L$, and the largest vector sublattice of $E$ containing $L$ in which $L$ is nestedly dense.

Proof It is easy to verify that $M$ is a vector subspace of $E$. If $x \in M, u, v \in L$ and $u \leqslant x \leqslant v$, then $u^{+} \leqslant x^{+} \leqslant v^{+}, u^{+}, v^{+} \in L$, and $v^{+}-u^{+} \leqslant v-u$. It follows that $x^{+} \in M$ which proves that $M$ is a vector sublattice of $E$. Let $z \in E$ be such that for every zero neighborhood $U$ of zero in $E$ one can find $x, y \in M$ with $x \leqslant z \leqslant y$ and $y-x \in U$. Then there exist $u, v \in L$ such that $u \leqslant x, y \leqslant v$, and $x-u \in U$, $v-y \in U$. Thus $u \leqslant z \leqslant v$ and $v-u \in U+U$. It follows that $x \in M$.

The rest of the assertion is obvious.

Examples 4.2 Let $E$ be a normed vector lattice with a strong order unit $e$ whose norm is the Minkowski functional of the order interval $[-e, e]$. Then every dense vector sublattice $L$ of $E$ containing $e$ is nestedly dense in $E$. In fact, for every $x \in E$ and 
$\varepsilon>0$ there is $u \in L$ such that $\|x-u\| \leqslant \varepsilon$ or, equivalently, $u-\varepsilon e \leqslant x \leqslant u+\varepsilon e$, where both $u-\varepsilon e$ and $u+\varepsilon e$ are in $L$. Let us mention a few concrete cases.

For a set $S$ with an algebra $\mathcal{A}$ of its subsets, let $B(S)$ denote the Banach lattice of all bounded real-valued functions on $S$ with the supnorm. Next, let $B(S, \mathcal{A})$ denote the closure in $B(S)$ of its vector sublattice $B_{0}(S, \mathcal{A})$ consisting of $\mathcal{A}$-simple functions. Then $B_{0}(S, \mathcal{A})$ is nestedly dense in $B(S, \mathcal{A})$.

Likewise, if $(S, \Sigma, \mu)$ is a positive measure space, then the vector lattice of all $\Sigma$-simple functions is nestedly dense in the Banach lattice $L_{\infty}(\mu)$.

If $S$ is any topological space, then every dense vector sublattice of the Banach lattice $C(S)$ containing the constant functions is nestedly dense in $C(S)$. In particular, the vector lattice of all eventually constant real sequences is nestedly dense in the Banach lattice $c$ of all convergent real sequences.

Examples 4.3 Nestedly dense proper vector sublattices can also be exhibited in some TVL's that are not of the type considered above.

(a) Let $(S, \Sigma, \mu)$ be a positive measure space. Then the countably $\Sigma$-simple functions in $L_{p}(\mu)(0<p<\infty)$ constitute a vector sublattice which is nestedly dense in $L_{p}(\mu)$.

(b) For $E=\ell_{p}(0<p<\infty)$ or $E=c_{0}$, let $L$ be the vector sublattice of $E$ consisting of sequences $x=\left(\xi_{j}\right)$ with $\xi_{2 k-1}=\xi_{2 k}$ for all large enough $k$. Then $L$ is nestedly dense in $E$. For instance, if $E=\ell_{p}$ and $1 \leqslant p<\infty$, let $x=\left(\xi_{j}\right) \in \ell_{p}$ and take any $\varepsilon>0$. Choose $k_{0}$ so that $\sum_{j>2 k_{0}}\left|\xi_{j}\right|^{p}<\varepsilon^{p} / 2^{p}$, and define $v=\left(v_{j}\right)$ by $v_{j}=\xi_{j}$ if $j \leqslant 2 k_{0}$, and $v_{2 k-1}=v_{2 k}=\max \left\{\xi_{2 k-1}, \xi_{2 k}\right\}$ if $k>k_{0}$. Then $v \in L$, $x \leqslant v$, and $\|v-x\|_{p} p \leqslant 2^{p} \sum_{k>k_{0}}\left(\left|\xi_{2 k-1}\right|^{p}+\left|\xi_{2 k}\right|^{p}\right)<\varepsilon^{p}$.

Question 4.4 Does every infinite dimensional Banach (or $F$-) lattice have a nestedly dense proper vector sublattice? In particular, is it so in $C(K)$, where $K$ is any infinite compact space?

Theorem 4.5 Let $E$ be a TVL, L a nestedly dense vector sublattice in $E$, and $F$ a nestedly complete TVL. Then every nestedly continuous positive linear operator $T: L \rightarrow F$ can uniquely be extended to a nestedly continuous positive linear operator $\tilde{T}: E \rightarrow F$, and if $T$ is a lattice homomorphism, so is $\tilde{T}$. Moreover, if $T$ is continuous, so is $\tilde{T}$, and if $T$ is a homeomorphic embedding, so is $\tilde{T}$.

Proof Take any point $x \in E$ and any Cauchy nest $\left(\left[u_{\alpha}, v_{\alpha}\right]\right)_{\alpha \in A}$ in $E$ such that $u_{\alpha}, v_{\alpha} \in L$ and $u_{\alpha} \leqslant x \leqslant v_{\alpha}$ for each $\alpha$. Then the net $\left(\left[T u_{\alpha}, T v_{\alpha}\right]\right)_{\alpha \in A}$ is a Cauchy nest in $F$ which, by the nested completeness of $F$, has a limit. It is easy to see that the limit, let us call it $\tilde{T} x$, does not depend on the choice of the nest $\left(\left[u_{\alpha}, v_{\alpha}\right]\right)$.

It is not hard to verify that the operator $\tilde{T}: E \rightarrow F$ thus obtained is additive and positively homogeneous, hence it is linear. Moreover, if $0 \leqslant x \in E$, then one may choose the intervals $\left[u_{\alpha}, v_{\alpha}\right]$ above so that $u_{\alpha} \geqslant 0$ for each $\alpha$. Then also $T u_{\alpha} \geqslant 0$ and, consequently, $\tilde{T} x \geqslant 0$. Thus $\tilde{T}$ is a positive operator.

Consider now any net $\left(x_{\alpha}\right)_{\alpha \in A}$ in $E$ such that $0 \leqslant x_{\alpha} \downarrow$ and $x_{\alpha} \rightarrow 0$. We are going to show that then $\tilde{T} x_{\alpha} \rightarrow 0$. Let $\mathcal{U}$ be a base of solid neighborhoods of zero in $E$. For every $\alpha \in A$ and $U \in \mathcal{U}$ choose $v_{\alpha, U} \in L$ so that $x_{\alpha} \leqslant v_{\alpha, U}$ and $v_{\alpha, U}-x_{\alpha} \in U$. Denote by $B$ the set of all 'indices' $\beta=\left(\alpha_{1}, \ldots, \alpha_{n} ; U_{1}, \ldots, U_{n}\right)$, where $\alpha_{1}, \ldots, \alpha_{n} \in A$ 
and $U_{1}, \ldots, U_{n} \in \mathcal{U}(n \in \mathbb{N})$. Endowed with a natural ordering, $B$ becomes a directed upward set. For each index $\beta$ of the above form, let $w_{\beta}=v_{\alpha_{1}, U_{1}} \wedge \cdots v_{\alpha_{n}, U_{n}}$. Then $w_{\beta} \in L$ and $0 \leqslant w_{\beta} \downarrow$. Given any $U \in \mathcal{U}$, choose $V \in \mathcal{U}$ with $V+V \subset U$ and $\alpha \in A$ so that $x_{\alpha} \in V$. Since $x_{\alpha}-v_{\alpha, V} \in V$, it follows that $v_{\alpha, V} \in V+V \subset U$. If $(\alpha, V) \leqslant \beta$ (that is, $\alpha=\alpha_{i}$ for some $i$ and $V=U_{j}$ for some $j$ ), then $w_{\beta} \leqslant v_{\alpha, V}$ and, consequently, $w_{\beta} \in U$. This proves that $w_{\beta} \rightarrow 0$. Therefore, also $T w_{\beta} \rightarrow 0$. Let $W$ be a solid neighborhood of zero in $F$, and choose $\beta$ (of the above form) so that $T w_{\beta} \in W$. Select $\alpha \in A$ so that $\alpha \geqslant \alpha_{1}, \ldots, \alpha_{n}$. Then $x_{\alpha} \leqslant x_{\alpha_{1}} \wedge \cdots \wedge x_{\alpha_{n}} \leqslant w_{\beta}$, hence $\tilde{T} x_{\alpha} \leqslant T w_{\beta}$ and so $\tilde{T} x_{\alpha} \in W$.

If $T$ is a lattice homomorphism, $x \in E$ and $u_{\alpha}, v_{\alpha} \in L$ are as in the beginning, then $u_{\alpha}^{+} \leqslant x^{+} \leqslant v_{\alpha}^{+}, v_{\alpha}^{+}-u_{\alpha}^{+} \leqslant v_{\alpha}-u_{\alpha} \rightarrow 0$, and $T u_{\alpha}^{+}=\left(T u_{\alpha}\right)^{+} \leqslant(\tilde{T} x)^{+} \leqslant$ $\left(T v_{\alpha}\right)^{+}=T v_{\alpha}^{+}$. It follows that $\tilde{T} x^{+}=(\tilde{T} x)^{+}$. Thus $\tilde{T}$ is a lattice homomorphism.

Let us now assume that $T$ is continuous, and verify also that $\tilde{T}$ is continuous. Let $W$ be a solid neighborhood of zero in $F$, and choose $U \in \mathcal{U}$ so that $T(U) \subset W$. Also, choose $V \in \mathcal{U}$ so that $V+V \subset U$. Now, let $x \in V$ and pick $u, v \in L$ so that $u \leqslant x \leqslant v$ and $v-u \in V$. Then $u, v \in U$, hence $T u, T v \in W$. Since $\tilde{T} x \leqslant T v$ and $-\tilde{T} x \leqslant-T u$, we have $|\tilde{T} x| \leqslant|T v|+|-T u| \in W+W$.

In the case $T$ is continuous an alternative argument could be used as well: From the general theory of TVL's it is known that then $T$ has a continuous positive linear extension $\tilde{T}: E \rightarrow \tilde{F}$, where $\tilde{F}$ is the completion of $F$, and that if $T$ is a homomorphism, so is $\tilde{T}$. Given any $x \in E$, choose the nest $\left(\left[u_{\alpha}, v_{\alpha}\right]\right)$ as in the first paragraph. Then observe that $\tilde{T} x$ must coincide with the unique point in the intersection of the intervals $\left[T u_{\alpha}, T v_{\alpha}\right]$ in $F$ so that $\tilde{T} x \in F$.

The last assertion follows from a general fact: If $E, F$ are TVs's, $L$ is a dense subspace of $E, T: L \rightarrow F$ is a linear homeomorphic embedding that admits a continuous linear extension to an operator $\tilde{T}: E \rightarrow F$, then also $\tilde{T}$ is a homeomorphic embedding. (This can easily be deduced from [8, Th. 3.4.2].)

Remark 4.6 Of course, if $E$ is metrizable, $E=(E,\|\cdot\|)$, then one may use $\sigma$-nests and sequences instead of nests and nets in the above proof. Moreover, in this case the argument showing that $\tilde{T}$ is $(\sigma$-)nestedly continuous can greatly be simplified as follows: Let $0 \leqslant x_{n} \in E, x_{n} \downarrow$ and $\left\|x_{n}\right\| \rightarrow 0$. For every $n$ there is $v_{n} \in L$ with $x_{n} \leqslant v_{n}$ and $\left\|v_{n}\right\| \leqslant\left\|x_{n}\right\|+\frac{1}{n}$, and by replacing $v_{n}$ with $v_{1} \wedge \cdots \wedge v_{n}$ we may assume that $v_{n} \downarrow$. Then $0 \leqslant \tilde{T} x_{n} \leqslant T v_{n}$ and $T v_{n} \rightarrow 0$ so that also $\tilde{T} x_{n} \rightarrow 0$.

Corollary 4.7 Let L be a metrizable TVL which is nestedly dense in its completion $\tilde{L}$, and let $F$ be any TVL. Then every nestedly continuous positive linear operator $T$ : $L \rightarrow F$ is continuous.

Proof The extension $\tilde{T}: E=\tilde{L} \rightarrow F$ of $T$ provided by the theorem is a positive linear operator, and $E$ is an $F$-lattice. Apply [2, Th. 5.19] to see that $\tilde{T}$ (hence $T$ as well) is continuous.

Example 4.8 Let $L=c_{00}$, the vector lattice of all eventually zero real sequences equipped with the supnorm. Then $L$ is nestedly closed, hence not nestedly dense in $\tilde{L}=c_{0}$, and the linear functional $f$ defined on $L$ by $f(x)=\sum_{j} \xi_{j}$ for $x=\left(\xi_{j}\right) \in L$ is positive and nestedly continuous but not continuous. 
For a TVL $L$, by a nested completion of $L$ we shall mean any nestedly complete TVL $M$ containing $L$ as a nestedly dense vector sublattice. Clearly, if $\tilde{L}$ is the (usual) completion of $L$, then the nested closure of $L$ in $\tilde{L}$ is a nested completion of $L$. The uniqueness, up to order-topological isomorphism, of a nested completion of $L$ follows from the following.

Proposition 4.9 Suppose that a TVL $L$ is nestedly dense in either of the nestedly complete TVL's $M$ and $N$. Then $M$ and $N$ are order-topologically isomorphic.

Proof From Theorem 4.5 it follows that if $T$ denotes the identity map from $L$ in $M$ to $L$ in $N$, then $T$ can be extended to an order-topological isomorphism from $M$ into $N$, and $T^{-1}$ can be extended to a like isomorphism from $N$ into $M$. It is easily seen that both these extended maps have to be onto.

Question 4.10 Which metrizable vector lattices $L$ are nestedly dense in their completion $\tilde{L}$ ? (Of course, it is so iff the nested completion of $L$ coincides with $\tilde{L}$.)

\section{Vector lattices $C(S)$ with non-complete order intervals}

In [10, p. 46], one can find an example of a normed Dedekind complete vector lattice, thus nestedly complete, which admits no intervally complete monotone norm. In this section, we give examples of normed lattices of type $C(S)$ (even for a compact space $S$ ) that are nestedly complete but are neither $\sigma$-Dedekind complete nor intervally complete, even in a very strong sense that no nontrivial order interval is complete.

All topological spaces occurring below are assumed to be Hausdorff, and we refer the reader to [6] for any unexplained topological notions or facts.

For a topological space $S$, we denote by $\mathcal{C}(S)$ the vector lattice of all continuous real-valued functions on $S$, and let $C(S)$ stand for its ideal consisting of bounded functions. If $A$ is a subset of $S$, then $\tau_{p}(A)$ denotes the topology in $\mathcal{C}(S)$ or $C(S)$ of pointwise convergence on $A$, and $\tau_{u}(A)$ that of uniform convergence on $A$. We write simply $\tau_{p}$ and $\tau_{u}$ when $A=S$; recall that $\tau_{u}$ in $C(S)$ is defined by the supnorm $\|\cdot\|_{\infty}$ which makes $C(S)$ a Banach lattice. In general, if $\|\cdot\|$ is a (semi)norm in $C(S)$, we denote by $\tau(\|\cdot\|)$ the topology defined by $\|\cdot\|$.

Proposition 5.1 For every topological space $S$, the vector lattice $\mathcal{C}(S)$ is nestedly complete in the topology $\tau_{p}$, and in any locally solid topology $\tau \geqslant \tau_{p}$. The same is true for every ideal in $\mathcal{C}(S)$, in particular, $C(S)$.

Proof Let $\left(\left[f_{\alpha}, g_{\alpha}\right]\right)$ be a Cauchy nest in in $\mathcal{C}(S)$. Then $f_{\alpha} \uparrow, g_{\alpha} \downarrow$ and $g_{\alpha}-f_{\alpha} \rightarrow 0$ $\left(\tau_{p}\right)$. It follows that the function $h$ defined on $S$ by $h(s)=\sup _{\alpha} f_{\alpha}(s)=\inf _{\alpha} g_{\alpha}(s)$ $(s \in S)$ is both lower and upper semicontinuous, hence continuous (cf. [9, Sec. 6.3]). Clearly, $h \in\left[f_{\alpha}, g_{\alpha}\right]$ for every $\alpha$.

Note that any locally solid topology $\tau$ in $C(S)$ is always weaker than $\tau_{u}$. This follows, e.g., from the fact that the order interval $\left[-1_{S}, 1_{S}\right]$ in $C(S)$, which is the closed unit ball for $\|\cdot\|_{\infty}$, is $\tau$-bounded. ( $1_{S}$ is the constant one function on $S$.)

We proceed with some relevant examples. 
Examples 5.2 If $D$ is a dense subset of $S$, then also the topology $\tau_{p}(D) \leqslant \tau_{p}$ is Hausdorff and locally solid. However, $\left(C(S), \tau_{p}(D)\right)$ need not be nestedly complete, though sometimes it is.

(a) To see this, let $S=[-1,1]$ and $D=[-1,1] \backslash\{0\}$. Next, for each $n \in \mathbb{N}$ let $f_{n}, g_{n} \in C[-1,1]$ be piecewise linear functions such that the graph of $f_{n}$ has vertices at the points $(-1,0),(0,0),\left(\frac{1}{n}, 1\right)$, and $(1,1)$, while that of $g_{n}$ has vertices at the points $(-1,0),\left(-\frac{1}{n}, 0\right),(0,1)$, and $(1,1)$. Then the order intervals $\left[f_{n}, g_{n}\right]$ in $C[-1,1]$ form a $\sigma$-nest and $g_{n}-f_{n} \rightarrow 0\left(\tau_{p}(D)\right)$. However, there is no $h \in C[-1,1]$ with $f_{n} \leqslant h \leqslant g_{n}$ for every $n$.

(b) If $S$ is completely regular, one may identify $C(S)$ with $C(\beta S)$ via the map $f \rightarrow \tilde{f}$, where $\tilde{f}$ is the unique continuous extension of $f$ to $\beta S$. Obviously, $C(\beta S)$ is nestedly complete for the topology $\tau_{p}(S)$ which is strictly weaker than $\tau_{p}(\beta S)$ when $S$ is noncompact.

Example 5.3 A normed lattice $C(S)$ with a non-complete order interval.

Let $S=[0,1]$, and $S_{n}=\left[1-\frac{1}{n}, 1-\frac{1}{n+1}\right]$ for $n=1,2, \ldots$ Define a monotone norm $\|\cdot\|$ in $C(S)$ by $\|f\|=\sum_{n=1}^{\infty} 2^{-n} \sup \left\{|f(s)|: s \in S_{n}\right\}+|f(1)|$. Clearly, $\|\cdot\| \leqslant$ $2\|\cdot\|_{\infty}$; on the other hand, $\tau(\|\cdot\|) \geqslant \tau_{p}$ so that the normed lattice $E=(C(S),\|\cdot\|)$ is nestedly complete. However, it fails to be intervally complete. In fact, let $h_{n}(t)=t^{n}$ for $t \in S(n \in \mathbb{N})$. Then the sequence $\left(h_{n}\right) \subset\left[0,1_{S}\right]$ is Cauchy but has no limit in $E$. Thus the order interval $\left[0,1_{S}\right]$ is not complete.

Example 5.4 A normed lattice $C(S)$ with no complete nontrivial order intervals.

Let $S=\left\{s_{1}, s_{2}, \ldots\right\}$ be a countable metric space without isolated points (e.g., $S=\mathbb{Q} \cap(0,1)$ with its natural metric). For $t \in S$ and $\varepsilon>0$, let $K(t, \varepsilon)$ denote the open ball in $S$ centered at $t$ and with radius $\varepsilon$. Define a monotone norm $\|\cdot\|_{1}$ in $C(S)$ by $\|f\|_{1}=\sum_{k=1}^{\infty} 2^{-n}\left|f\left(s_{n}\right)\right|$. Then $\|\cdot\|_{1} \leqslant\|\cdot\|_{\infty}$ and, on the other hand, $\tau\left(\|\cdot\|_{1}\right) \geqslant \tau_{p}$. Moreover, $\tau\left(\|\cdot\|_{1}\right)=\tau_{p}$ on every order interval in $C(S)$, and the normed lattice $E=\left(C(S),\|\cdot\|_{1}\right)$ is nestedly complete. However, no nontrivial order interval in $E$ is norm complete. To see this, let $0 \leqslant g \in E, g \neq 0$. Then there exists a ball $K(t, \varepsilon)$ in $S$ and a constant $c>0$ such that $g(s)>c$ for all $s \in K(t, \varepsilon)$. For each $n \in \mathbb{N}$, let $\varepsilon_{n}=\varepsilon / n$ and let $h_{n}: S \rightarrow[0, c]$ be a continuous function such that $h_{n}(t)=c$ and $h_{n}(s)=0$ for $s \in S \backslash K\left(t, \varepsilon_{n}\right)$. Then $\left(h_{n}\right)$ is a sequence in the order interval $[0, g]$ which converges pointwise to $1_{\{t\}}$. It follows that $\left(h_{n}\right)$ is Cauchy in $E$ but has no limit.

Note that, by identifying $C(S)$ with $C(\beta S)$ (see Example 5.2(b)), one can get a normed lattice $C(K)$ with the required property for a compact space $K$.

Finally, it may be worth observing that if a measure $\mu$ is defined on all subsets of $S$ so that $\mu\left(\left\{s_{n}\right\}\right)=2^{-n}(n \in \mathbb{N})$, then $\|f\|_{1}=\int_{S}|f| d \mu$ for $f \in C(S)$. Then, moreover, $C(S)$ is dense in $L_{1}(\mu)$, and $L_{1}(\mu)$ is order-isometric with $\ell_{1}$.

We finish this section with two general results concerning the existence of complete nontrivial order intervals in $C(S)$ lattices. In the proof of the first one, we will need the following.

Lemma 5.5 Let $S$ be a regular topological space and $\left(s_{n}\right)$ a sequence of distinct points in $S$. Then there exists a subsequence $\left(t_{n}\right)$ of $\left(s_{n}\right)$ and a sequence of disjoint open sets $\left(U_{n}\right)$ in $S$ such that $t_{n} \in U_{n}$ for every $n$. 
Proof This is fairly easy to prove when the sequence $\left(s_{n}\right)$ has a convergent subsequence. Assume, therefore, that this is not the case. Then for every point $s \in S$ and any infinite subset $N$ of $\mathbb{N}$ there exists a closed neighborhood $V$ of $s$ such that $s_{n} \notin V$ for infinitely many $n \in N$.

Now, start with $n_{1}=1, t_{1}=s_{n_{1}}$, and $N_{1}=\mathbb{N}$, and choose a closed neighborhood $V_{1}$ of $t_{1}$ such that the set $N_{2}:=\left\{n \in N_{1}: s_{n} \notin V_{1}\right\}$ is infinite. Next, denote $n_{2}=\min N_{2}, t_{2}=s_{n_{2}}$, and choose a closed neighborhood $V_{2}$ of $t_{2}$ which is disjoint from $V_{1}$ and such that the set $N_{3}:=\left\{n \in N_{2}: s_{n} \notin V_{2}\right\}$ is infinite. Proceed in this manner to obtain required sequences $\left(t_{n}\right)$ and $\left(U_{n}\right):=\left(\right.$ Int $\left.V_{n}\right)$.

Theorem 5.6 Let $S$ be a compact space, and $\|\cdot\|$ a monotone norm in $C(S)$ such that $\tau(\|\cdot\|) \geqslant \tau_{p}$. Then the order interval $\left[0,1_{S}\right]$ in $C(S)$ is $\|\cdot\|$-complete (and so are all the other order intervals in $C(S))$ iff $\|\cdot\|$ is equivalent to $\|\cdot\|_{\infty}$.

Proof There is nothing to prove when $\|\cdot\|$ and $\|\cdot\|_{\infty}$ are equivalent.

Suppose, therefore, that $\left[0,1_{S}\right]$ is $\|\cdot\|$-complete, but the two norms in question are not equivalent. Then, as easily seen, for every $c>0$ there is $0 \neq h \in\left[0,1_{S}\right]$ such that $\|h\| \leqslant c\|h\|_{\infty}$. It follows that there exists a sequence $\left(h_{n}\right)$ in $\left[0,1_{S}\right]$ such that $\left\|h_{n}\right\|_{\infty}=1$ and $\left\|h_{n}\right\| \leqslant 2^{-n}$ for each $n$. For every $n$ choose a point $s_{n} \in S$ so that $h_{n}\left(s_{n}\right)=1$. By passing to a suitable subsequence, and relabelling, we arrive to one of the following two cases.

Case 1 The sequence $\left(s_{n}\right)$ is constant, say $s_{n}=s_{0}$ for each $n$. Then, as $\left\|h_{n}\right\| \rightarrow 0$, also $1=h_{n}\left(s_{n}\right)=h_{n}\left(s_{0}\right) \rightarrow 0$; a contradiction.

Case 2 The sequence $\left(s_{n}\right)$ has pairwise distinct terms. In view of the lemma above, by passing to a subsequence we may assume that there is a sequence $\left(U_{n}\right)$ of disjoint open sets such that $s_{n} \in U_{n}$ for each $n$. Let $s_{0}$ be an accumulation point of $\left(s_{n}\right)$. Clearly, we may assume that $s_{n} \neq s_{0}$ and $s_{0} \notin U_{n}$ for each $n$. For each $n$, choose a continuous function $g_{n}: S \rightarrow[0,1]$ with $g_{n}\left(s_{n}\right)=1$ and $g_{n}(s)=0$ for $s \notin U_{n}$, and let $f_{n}=h_{n} \wedge g_{n}$. Then $f_{n} \in\left[0,1_{S}\right], f_{n}\left(s_{n}\right)=1,\left\|f_{n}\right\| \leqslant\left\|h_{n}\right\| \leqslant 2^{-n}$, and $f_{m} \wedge f_{n}=0$ whenever $m \neq n$. It follows that the series $\sum_{n} f_{n}$ is $\|\cdot\|$-Cauchy, and that all its partial sums are in $\left[0,1_{S}\right]$. Therefore, it is $\|\cdot\|$-convergent, hence also pointwise convergent, to some $f \in\left[0,1_{S}\right]$. But $f\left(s_{n}\right)=1$ for each $n$, while $f\left(s_{0}\right)=0$, which is clearly impossible.

For a topological space $S$ and an open set $V \subset S$, we denote by $C_{0}(S \| S \backslash V)$ the ideal in $C(S)$ consisting of those functions that vanish on $S \backslash V$ (see [9, 4.1.3] for this notation). Note that $C_{0}(S \| S \backslash V)$ is $\tau_{u}$-closed, hence $\tau_{u}$-complete.

We recall that a Baire topological space is one in which the intersection of any sequence of dense open sets is dense (see [7]). Classic examples of Baire spaces include complete metric spaces, compact spaces, and locally compact spaces.

Theorem 5.7 Let $S$ be a Baire topological space, and $D$ a dense subset of $S$ such that $S \backslash D$ is nowhere dense. Furthermore, let $\tau$ be a metrizable vector topology in $C(S)$ which is stronger than $\tau_{p}(D)$. Then every nonempty open set $U$ in $S$ contains a nonempty open set $V$ such that $\tau \geqslant \tau_{u}(V)$.

In consequence, $\tau \geqslant \tau_{u}$ on the ideal $C_{0}(S \| S \backslash V)$. If, moreover, $\tau$ is locally solid (so that $\left.\tau \leqslant \tau_{u}\right)$, then $\tau=\tau_{u}$ on $C_{0}(S \| S \backslash V)$. 
Proof Let $\tau^{c}$ denote the strongest locally convex topology in $C(S)$ that is coarser than $\tau$. A base of $\tau^{c}$-neighborhoods of zero can be obtained by taking convex hulls of $\tau$-neighborhoods of zero. In consequence, $\tau^{c}$ is metrizable and $\tau_{p}(D) \leqslant \tau^{c} \leqslant \tau$. Therefore, it is clear that it is enough to prove the main assertion of the theorem for the topology $\tau^{c}$. Thus without loss of generality we may simply assume that $\tau$ itself is locally convex.

Let $\left(\|\cdot\|_{m}\right)$ be an increasing sequence of seminorms defining $\tau$. Take any nonempty open subset $U$ of $S$ and denote $R=S \backslash D$. Since Int $\bar{R}=\emptyset$, also the set $U \backslash \bar{R}$ is open and nonempty, hence Baire. Now, for every $m, n \in \mathbb{N}$, let

$$
F_{m, n}=\left\{s \in U \backslash \bar{R}:|f(s)| \leqslant n\|f\|_{m}, \forall f \in C(S)\right\} .
$$

Evidently, each of these sets is closed in $U \backslash \bar{R}$, and they cover all of $U \backslash \bar{R}$ because $\tau \geqslant \tau_{p}(D)$. By the Baire property of $U \backslash \bar{R}$, there is a pair $m, n$ for which $V:=$ Int $F_{m, n} \neq \emptyset$. Since $\tau_{u}(V) \leqslant \tau\left(\|\cdot\|_{m}\right) \leqslant \tau, V$ is as required.

Corollary 5.8 Let $S$ be a completely regular Baire topological space, and D a dense subset of $S$ such that $S \backslash D$ is nowhere dense. Furthermore, let $\tau \geqslant \tau_{p}(D)$ be a metrizable locally solid topology in $C(S)$. Then every nontrivial order interval in $C(S)$ contains a nontrivial order interval on which $\tau=\tau_{u}$; consequently, the latter interval is $\tau$-complete.

Proof Obviously, it suffices to prove this for order intervals of type $[0, f]$. We may assume that $\|f\|_{\infty}>1$ so that the set $U=\{s \in S: f(s)>1\}$ is nonempty and open in $S$. By the theorem, there is a nonempty open set $V \subset U$ such that $\tau=\tau_{u}$ on the ideal $C_{0}(S \| S \backslash V)$. By the complete regularity of $S$, there is a continuous function $g: S \rightarrow[0,1]$ such that $g\left(s_{0}\right)=1$ for some $s_{0} \in V$, and $g(s)=0$ for all $s \notin V$. Then the order interval $[0, g]$ is as required.

Acknowledgments I am very grateful to Professor Witold Wnuk for his interest in this paper and many useful comments and references. I would also like to thank the referee for a number of corrections and, especially, suggestions that led me to some improvements, in particular, to the present form of Theorem 3.4.

Open Access This article is distributed under the terms of the Creative Commons Attribution Noncommercial License which permits any noncommercial use, distribution, and reproduction in any medium, provided the original author(s) and source are credited.

\section{References}

1. Abramovich, J.A.: Some criteria for the interval completeness of normed lattices (Russian), Izv. Vysš. Učebn. Zaved. Matematika, 6(169), 3-8 (1976). [English translation: Soviet Math. (Iz. VUZ) 20(6), 1-5 (1976).]

2. Aliprantis, C.D., Burkinshaw, O.: Locally Solid Riesz Spaces with Applications to Economics, 2nd edn. Math. Surveys and Monographs, vol. 105, Amer. Math. Soc., (2003)

3. Aliprantis, C.D., Burkinshaw, O.: Positive Operators, 2nd edn. Springer, Berlin (2006)

4. Castillo, J.M.F., González, M.: Three-space problems in Banach Space Theory, pp. xii+ 267. Lecture Notes Math., vol. 1667. Springer, Berlin (1997)

5. Drewnowski, L., Nawrocki, M.: Connectedness in some topological vector-lattice groups of sequences, Math. Scand. (to appear) (2010)

6. Engelking, R.: General Topology. PWN_Polish Sci. Publ., Warszawa (1977) 
7. Haworth, R.C., McCoy, R.A.: Baire spaces. Dissert. Math. 141, 77 (1977)

8. Jarchow, H.: Locally Convex Spaces. Teubner, Stuttgart (1981)

9. Semadeni, Z.: Banach Spaces of Continuous Functions. PWN-Polish Sci. Publ., Warszawa (1971)

10. Veksler, A.I.: Tests for interval completeness and interval-complete normability of KN-lineals, Izv. Vysš. Učebn. Zaved. Matematika 4(95), 36-46 (1970) (in Russian)

11. Zaanen, A.C.: Riesz Spaces II. North-Holland, Amsterdam (1983) 